American J. of Engineering and Applied Sciences 4 (4): 470-476, 2011

ISSN 1941-7020

(C) 2014 S. Gruber et al., This open access article is distributed under a Creative Commons Attribution

(CC-BY) 3.0 license

\title{
A Wireless Sensor Node Supporting Various Powers Supply and Communication Methods
}

\author{
${ }^{1,2}$ Stefan Gruber, ${ }^{2}$ Hannes Reinisch, ${ }^{2}$ Hartwig Unterassinger, \\ ${ }^{1,2}$ Martin Wiessflecker, ${ }^{1}$ Gunter Hofer, \\ ${ }^{1,2}$ Michael Klamminger, ${ }^{2}$ Wolfgang Pribyl and ${ }^{1}$ Gerald Holweg \\ ${ }^{1}$ Development Center Graz, \\ Infineon Technologies Austria AG, Babenberg Straße 10, 8020, Graz \\ ${ }^{2}$ Institute of Electronics, Graz University of Technology, Inffeldgasse 12, 8010, Graz
}

\begin{abstract}
Problem statement: This study presents a wireless sensor node dedicated for in-tire pressure and temperature monitoring. Additional features set it apart from the many different systems available on the highly competitive market. State-of-the-art implementations are mounted on the rim of a tire. Here the sensor node will be mounted on the inner liner of the tire. By moving the sensor node from the rim to the inner liner, new challenges in the analog design arise. Approach: To implement this sensor node new power supply methods are investigated. Next, analog designs with the focus on low power are developed. Since the sensor node is operated in a harsh environment, robustness is a key issue. Results: The discussed sensor is capable of either active or passive communication. The passive communication is used in the field of Radio Frequency Identification (RFID), whereas active communication is used to transmit the sensor data. A combination of both is also possible. To supply the chip in the tire an RF energy harvesting interface is implemented. An input sensitivity of $-19.7 \mathrm{dBm}$ for the harvesting interface and $-12.5 \mathrm{dBm}$ for the RFID interface is measured. The on-chip temperature sensor consumes $4 \mu \mathrm{A}$ including the analog to digital converter. Conclusion: Choosing the discussed sensor node for in-tire monitoring enables new fields of applications. With this sensor node sensing additional parameters like road condition is possible. Using passive communication improves warehouse management for tire manufactures. Also, tire type identification at the car is enabled.
\end{abstract}

Key words: Tire pressure monitoring system, radio frequency identification, energy harvesting, AntiLock Braking System (ABS), Electronic Stability Control (ESC), Integrated Circuit (IC), RFID interface, inner liner

\section{INTRODUCTION}

Modern cars and trucks are equipped with Tire Pressure Monitoring Systems (TPMS). These systems trigger an alarm if the pressure in one or more of the tires is too low. In the United States of America TPMS is already mandatory for new cars. The European Commission also announced to make TPMS mandatory for all new cars by 2012. TPMS improve the safety and reduce $\mathrm{CO}_{2}$ emissions caused by increased fuel consumption due to under inflation. By now two different systems can be distinguished: (1) Indirect systems do not require dedicated sensors. Parameters extracted from the Anti-Lock Braking System (ABS) and the Electronic Stability Control (ESC) are used to estimate the pressure in the tires. The main advantage of this approach is that it is inexpensive and easy to implement. The required calibration of course is a major drawback. (2) Direct systems use dedicated sensors in the tires to measure the pressure directly. The data is transmitted using an active transmitter. Direct systems are more expensive than indirect systems. But providing additional features makes them advantageous compared to indirect systems.

As discussed in (Flatscher et al., 2009), state-ofthe-art sensors used for TPMS are mounted on the rim of a tire. If the chip can be attached to the inner liner of the tire, additional parameters can be sensed (e.g., road condition, or tire temperature). So the design goal for the presented Integrated Circuit (IC) is to make it suitable for inner liner attachment.

In order to increase attractiveness of the IC for the tire manufacturers and consumers it is equipped with Radio Frequency Identification (RFID)

Corresponding Author: Stefan Gruber, Development Center Graz, Infineon Technologies Austria AG, Babenberger Straße 10, 8020, Graz 
Am. J. Engg. \& Applied Sci., 4 (4): 470-476, 2011

technology, which allows simple warehouse and supply chain management. Additionally, tire type identification is possible in the car. Providing tire parameters like tread pattern or rubber type can improve ABS and ESC performance.

This study presents a wireless sensor node dedicated for direct TPMS. Additionally, this IC is equipped with RFID technology. This study is a continuation of the study presented in (Gruber et al., 2010) and is organized as follows: After a short introduction to the architecture, the possible power sources are introduced. A detailed discussion of the different analog blocks follows. The study concludes by presenting different application scenarios.

As discussed, the IC is mounted on the inner liner of a tire. Figure 1 shows the simplified cross section of a tire including the mounting position of the IC. Because of the limitations in weight, size and mechanical stress, the IC cannot be powered by a battery if it is mounted on the inner liner of the tire (Flatscher et al., 2009). Instead, an energy harvesting system is employed. The harvester transforms electromagnetic waves emitted by a base station in the car to DC power.

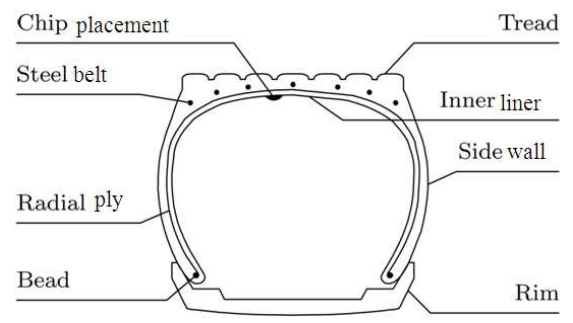

Fig. 1: Cross section of a tire including the expected mounting position of the sensor node

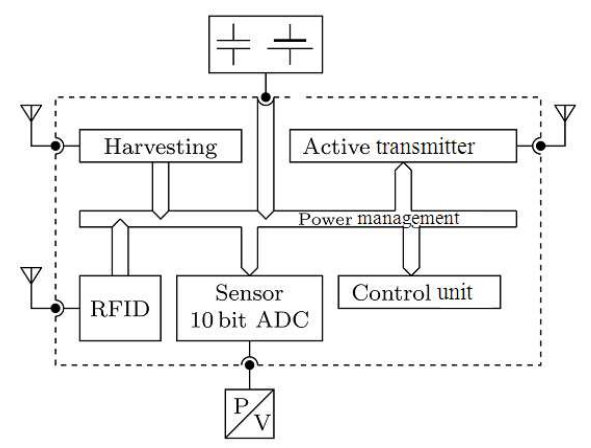

Fig. 2: Simplified block diagram of the wireless sensor node
The IC can be divided into six different blocks:

- Control unit

- Power source (energy harvesting)

- RFID

- Sensor and ADC

- Active transmitter

- Power management

The simplified block diagram is shown in Fig. 2; all the blocks are discussed in the following chapters in more detail.

\section{MATERIALS AND METHODS}

Moving the chip from the rim to the inner liner of the tire leads to new design challenges. The main limitation is the available power. Each block has to be as low in power consumption as possible. Of course, limiting the current consumption results in more noise and less bandwidth. So a trade-off between current consumption and robustness has to be found. For this design nominal bias currents from 1-10 nA are used. Conventional analog designs require large resistors and transistors to generate such low currents. So new design concepts, like switched capacitor approaches, have to be found. Reducing the current also leads to high ohmic nodes which are sensitive to cross-talk.

So not only the design is crucial but also the layout becomes very important. The next few chapters give an overview of the chip design.

Power sources: Depending on the application, the chip can be powered by three different power sources: (1) if the IC is used for TPMS, an electromagnetic energy harvesting system is employed. The energy gained by this system is stored in an off-chip buffer capacitor. (2) In the case of using RFID, the power is gained by the RFID interface. (3) One of the off-chip capacitors $\left(\mathrm{C}_{\mathrm{Buffer} 1}\right.$ or $\left.\mathrm{C}_{\mathrm{Buffer} 2}\right)$ of the harvesting interface can simply be replaced by a battery. Generally, any DC source can be used to replace one of the buffer capacitors. The DC/DC charge pump in the harvesting unit generates the required voltage level for low voltage inputs. Measurements using a small photovoltaic cell to power the IC have shown satisfactory results.

The analog front-end used as energy harvesting interface is presented in (Reinisch et al., 2010). As shown in Fig. 3 it is composed of two stages. The first stage is formed by a rectifier which converts the RF input into DC voltage. The converted energy is stored in a buffer capacitor. The second stage is a DC/DC 
charge pump. Using the charge pump, the voltage in the buffer capacitor is transformed to higher levels. An additional buffer capacitor is used to store the output energy of the second stage. If the voltage level in this buffer capacitor is high enough, the sensor is activated.

If the sensor node is powered by the RFID field, the analog front-end of the RFID interface transforms the RF energy to DC energy. This interface is first presented in (Missoni et al., 2008). It is operable in a frequency range from $1 \mathrm{MHz}-2.45 \mathrm{GHz}$. This enables supporting different frequency standards commonly used for RFID. The implemented protocols are EPC HF and EPC UHF. Depending on the input frequency the according protocol is chosen. In comparison to the harvesting interface the required input power is higher if the chip is powered by the RF field of the RFID reader. This is because the analog front-end of the RFID interface has modulation and demodulation capabilities.

The second buffer capacitor $\left(\mathrm{C}_{\mathrm{Buffer} 2}\right)$ of the harvesting interface can be replaced by a battery. In this case the sensor node is operating in polling mode, because the voltage level is always high enough to operate the sensor node. The sensor data is cyclically transmitted to the base station using the active transmitter. It is also possible to replace the first buffer capacitor $\left(\mathrm{C}_{\text {Bufferl }}\right)$ with a DC source. This is useful for low voltage sources, as the input voltage is amplified using the charge pump of the harvesting interface.

Control unit: To control the different analog blocks on the IC a state machine is implemented. Traditionally, such digital control units are implemented in synchronous logic. The behavior is described in a hardware description language, which is synthesized. Afterwards the layout is generated by powerful tools. For this sensor node a different approach is chosen. Driven by the strict power requirements, the state machine is implemented in asynchronous logic. The advantage of this approach is that no currents generated by clock signals are flowing. Especially, when only a few transitions are required compared to the clock frequency, this method saves power. The drawback is that formal verification of this approach is difficult.

The inputs of the state machine are logic signals generated by level detectors. These level detectors monitor the input voltage of the sensor node. Depending on these voltage levels different states are entered. Additionally, the output voltage levels of the different voltage regulators used for the power supply are monitored. If one of these voltage levels is too low, the operating mode is changed. If RFID mode is not considered, four different states are distinguished. The flow chart of the state machine is depicted in Fig. 4.

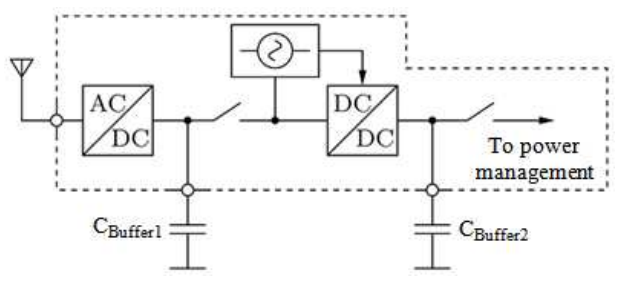

Fig. 3: Block diagram of the harvesting interface

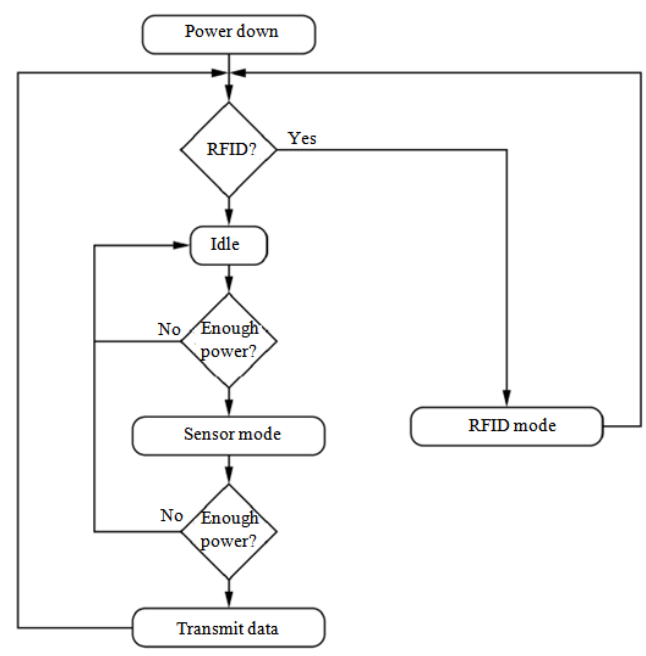

Fig. 4: Flow chart of the state machine used for controlling the sensor

For low input power the chip is turned off. Only the energy harvesting unit operates and stores some energy in the buffer capacitor. If the energy in the buffer capacitor is high enough, the sensor is started. After a successful operation the sensor data is stored in the on-chip memory. From now on the IC waits until the energy is high enough for an active transmission of the data. If for any reason the voltage at one of the voltage regulators of the power supply is too low, the chip is reset and the operation starts from the beginning.

If the IC is in RFID mode, the digital control unit of the RFID block takes over the control. The communication follows EPC (U) HF standards. By using memory write and read functions different blocks of the sensor node are controlled. Thus it is also possible to operate the on-chip sensor by the RFID interface.

To reduce leakage currents unused blocks are turned off completely. Thus in power-down state undefined signals are generated by these blocks. Additionally, during start-up wrong signals may be generated. To solve this problem an isolation-logic is implemented. This logic monitors the supply voltage of the blocks which may provide wrong or undefined 
signals. Only if the supply is high enough the output signals are forwarded to the control logic. Otherwise the isolation logic generates a defined signal.

Figure 5 shows the principle of the isolation logic. A comparator monitors the supply voltage (VDDSIN). The result of this comparison is gated using an AND gate with the signal (SIn). As long as the signal comp is at low level, the AND gate will not draw any current regardless the value of SIn. If different voltage supply levels appear, level shifters are required additionally.

Sensor and analog to digital converter: To measure the temperature an on-chip temperature sensor is implemented. It compares a temperature dependent to a temperature independent voltage. To generate these voltages a low power bias cell is implemented. The bias cell operates according to the principle of a switch capacitor band gap reference. This principle is first published in (Peng et al., 2009).

For sensing the pressure an off-chip MicroElectromechanical System (MEMS) device is necessary. To allow different types of this device an analog input at the presented wireless sensor node is available. The off-chip sensor feeds a voltage proportional to the pressure to the Analog to Digital Converter (ADC) on the wireless sensor node.

To gain a digital value of the temperature and the pressure a 10bit successive approximation ADC is implemented. The Digital to Analog Converter (DAC) is formed by a charge redistribution architecture using a capacitive split array. The comparator operates according to the time-domain comparator published in (Agnes et al., 2008). By using a clock frequency of $1 \mathrm{MHz} 36 \mathrm{kSamples} / \mathrm{s}$ are achieved.

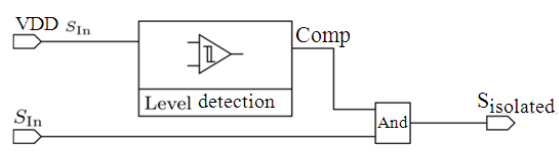

Fig. 5: Isolation logic to isolate undefined digital signals

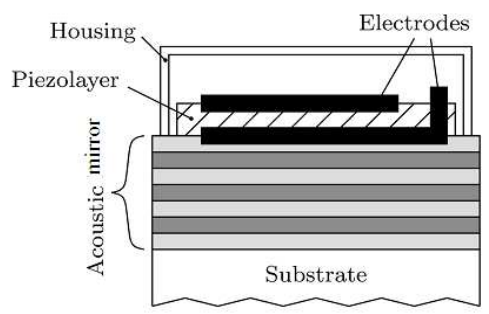

Fig. 6: Cross section of a bulk acoustic wave resonator
Active transmitter: The sensor data can be transmitted using an active transmitter. For the same reasons as for the battery, no off chip crystal can be used for the active transmitter. Instead, a Bulk Acoustic Wave (BAW) resonator is used as frequency reference. The cross section of a BAW is shown in Fig. 6. Referring to (Bi and Barber, 2008), the functionality of a BAW can be described as follows: The basic BAW resonators consist of a thin-film layer of piezoelectric material sandwiched between two metal thin-film electrodes. The voltage or the electrical field between the two electrodes excites an acoustic wave. The wave bounces back from the top and bottom surfaces of the two electrodes and an acoustic cavity is formed between the top surface of the upper electrode and the bottom surface of the lower electrode. The frequency at which the resonance occurs is determined by the thickness of the piezolayer and the thickness and mass of the electrodes.

Compared to a crystal, the BAW is more robust against mechanical stress. Additionally, the start-up time of oscillators using a BAW is in the range of only a few $\mu$ s, whereas crystal based oscillators need several ms. Thus, the energy consumption can be improved significantly.

The main drawback of the BAW technology is the temperature dependency of about $-19 \mathrm{ppm} / \mathrm{K}$ (Aigner, 2005) of the reference frequency. Thus, such resonators need temperature compensation. For the presented chip, the correction is done by mixing the reference frequency with a temperature dependent oscillator. The architecture is shown in Fig. 7. The current of the IQ oscillator is adjusted depending on the temperature.

This frequency is mixed with half of the frequency of the BAW resonator. The result is amplified and afterwards converted to a single-ended signal. By shifting the frequency of the IQ oscillator to different values, different transmission bands can be selected.

Power management: Caused by the large number of possible input voltage levels and input voltage slew rates, power management gets sophisticated. Due to power gating of the analog blocks more than ten different power rails can be identified on the IC. The power management ensures that the necessary voltage is available for specific blocks at a specified time. The maximum allowed voltage level for a regular MOS transistor in this process is $1.5 \mathrm{~V}$. As the input voltage can be more than twice as high, over-voltage protection is necessary.

As shown in Fig. 8, depending on the power supply available, the power is routed differently. If the chip is supplied by the RFID field, all necessary blocks are supplied by the RFID front-end. 
Am. J. Engg. \& Applied Sci., 4 (4): 470-476, 2011

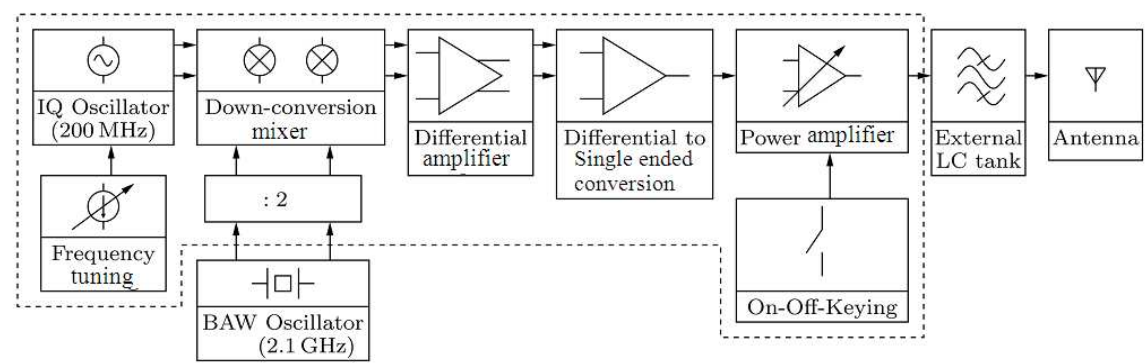

Fig. 7: Block diagram of the active transmitter

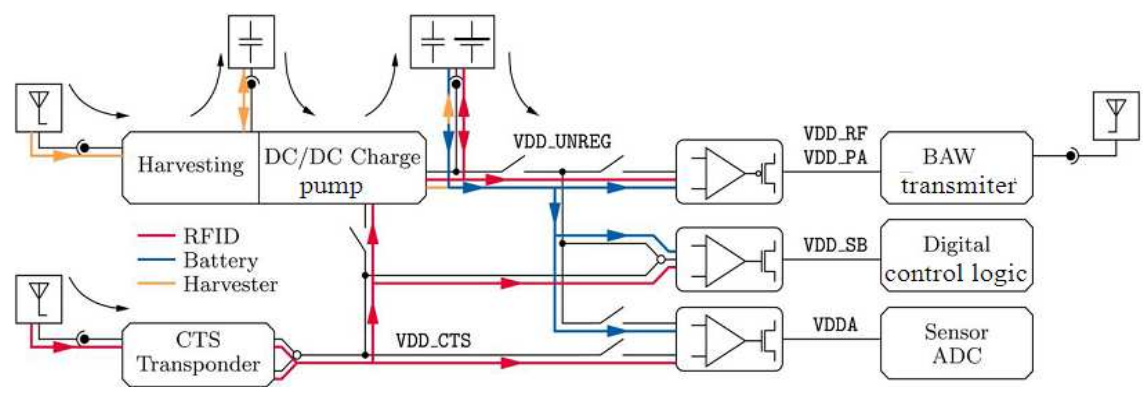

Fig. 8: Power flows on the sensor node depending on the used power supply

If the power provided by this interface is more than required to operate the IC, the additional energy is stored in the buffer capacitor using the DC/DC charge pump of the harvesting interface. This is necessary to operate the active transmitter because the power available from the RF field is not enough for a successful operation of the power amplifier. If the IC is supplied by the harvester, the energy is first stored in the buffer capacitor. Afterwards the chip is powered by the energy stored in this capacitor. By replacing this capacitor with a battery, all required blocks are powered by this device. As mentioned earlier, the buffer capacitor used for the DC/DC charge pump can also be replaced by an off chip low voltage DC source.

Four different regulator architectures, which provide the necessary supply voltages, are implemented on the IC. The architectures of the regulators are distinguished by the type of the pass device. NMOS types are chosen for the control logic and the sensor with the ADC. The active transmitter is supplied by two PMOS types. One regulator is used for the power amplifier and one is used for the carrier generation unit.

Generally, the stabilization of the NMOS types is easier than PMOS types. Thus, less current is necessary for the regulation circuitry of these types of regulators. The drawback is that low drop-out regulation is not possible without additional effort. The gate level of the
NMOS transistor needs to be at least one threshold voltage higher than the output voltage of the regulator. So, a charge pump is necessary which generates a voltage for the gate when the input voltage of the regulator is too low. To stabilize PMOS regulators an off-chip capacitor is often used. This capacitor forms a dominant pole. For the regulator used for the carrier generation unit a different approach is chosen. Here the dominant pole is formed inside the regulator. Although the regulator is stable, it has bad line regulation capabilities. But, as no fast transients during the operation of the PMOS regulator are expected, line regulation capabilities are not crucial. To deal with the transient at start-up a start-up control is implemented. The advantage of this method is that it makes an offchip capacitor unnecessary.

\section{RESULTS}

The implemented sensor node allows different application scenarios. Depending on the power supply and the communication method a different operating mode is selected. Figure 9 gives an overview of the different possible communication scenarios. The symbol holding the exclamation mark, which is the symbol used for TPMS failures, indicates the sensor. The sensor node can be operated as RFID tag. In this case it is also possible to use the on-chip sensor. 
Am. J. Engg. \& Applied Sci., 4 (4): 470-476, 2011

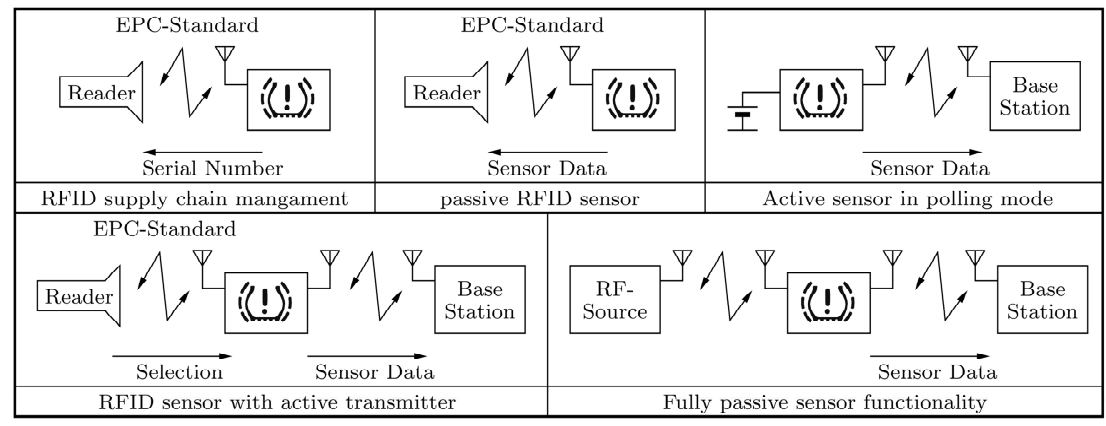

Fig. 9: Communication scenarios possible with the presented wireless sensor node

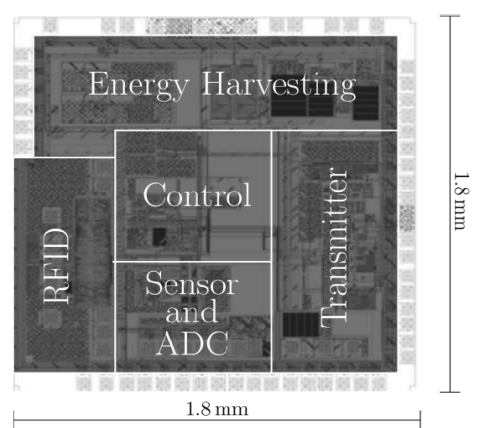

Fig. 10: Layout of the wireless sensor node

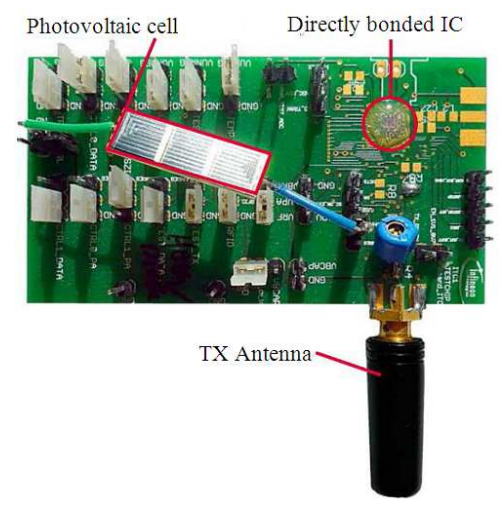

Fig. 11: Wireless sensor node powered by a photovoltaic cell

For transmitting the data either the passive backscattering or active transmission can be used. If the chip is supplied by a battery, it behaves like a general purpose wireless sensor node. The data is transmitted cyclically using the active transmitter. Instead of the battery the harvesting interface can be used. In this case the chip is supplied by an RF field. If the energy harvested is high enough, the sensor data is transmitted using the active transmitter.
The IC is fabricated in an Infineon $0.13 \mu \mathrm{m}$ CMOS process. Figure 10 shows its layout. As a lot of test pads are implemented, the size is pad limited. For testing and measurement purposes the IC is directly bonded on a printed circuit board. First measurement results are promising. Functional tests are finished. All discussed communications scenarios are successfully tested. As an application example, Fig. 11 shows the wireless sensor node configured as active transmitter and powered by a photovoltaic cell.

For performance evaluation, the key parameters of the different blocks are measured. The input sensitivity of the harvesting interface and the UHF RFID interface is -19.7 and $-12.5 \mathrm{dBm}$ respectively. As already explained, the harvesting interface requires no modulation and demodulation capabilities. That is why the input sensitivity of the RFID interface is larger. Additionally, the load current is about $10 \mu \mathrm{A}$, whereas in the harvesting case the load current is only 200nA. State-of-the-art UHF RFID tags, like NXP's UCODE, have a sensitivity below $-18 \mathrm{dBm}$. The main difference between these tags and the one presented here is the input bandwidth. The frontend here operates at either UHF or HF, while commercially available tags operate in UHF only.

The bias cell used for the temperature sensor has a measured current consumption of $500 \mathrm{nA}$ and the area consumption is a third compared to conventional designs. At a sampling rate of $36 \mathrm{kSamples} / \mathrm{s}$, the current consumption of the ADC converting the temperature value into a digital representative is only $4 \mu \mathrm{A}$. This current is mostly consumed by the buffers used to drive the DAC.

The power amplifier supports four different power levels: $-6.4,-1.5,4.0$ and $5.4 \mathrm{dBm}$. Thus, the power ranges of state-of-the-art TPMS modules are covered. The data is Manchester encoded and four different data rates are supported: $250,125,62.5$ and $31.25 \mathrm{kbit} \mathrm{sec}^{-1}$. 
Am. J. Engg. \& Applied Sci., 4 (4): 470-476, 2011

\section{DISCUSSION}

A comparable study is presented in (Flatscher et al., 2010). This study also presents a TPMS module for in-tire pressure monitoring. There are two distinctive differences: (1) The study in (Flatscher et al., 2010) makes use of a scavenging unit which converts vibration into electrical energy. By using an electromagnetic harvesting interface, as it is done in this study, no motion is required to power the sensor module. (2) This study increases the field of application by the RFID interface. Next to sensing functionality also identification is possible.

\section{CONCLUSION}

The study presented a wireless sensor node which enables different applications scenarios. Combining RFID technology with a TPMS module makes the technology attractive to tire manufacturers and car owners. Using a broadband RFID interface allows worldwide usage of the tag. The electromagnetic harvesting interface allows implementing a maintenance-free sensor node.

\section{ACKNOWLEDGEMENT}

This study has been partly funded by the Austrian Research Promotion Agency (FFG) within the project iTire.

\section{REFERENCES}

Agnes, A., E. Bonizzoni, P. Malcovati and F. Maloberti, 2008. A 9.4-ENOB $1 \mathrm{~V} 3.8 \mu \mathrm{W} 100 \mathrm{kS} / \mathrm{s}$ SAR ADC with time-domain comparator. Proceedings of the IEEE International Solid-State Circuits Conference, Feb. 3-7, IEEE Xplore Press, San Francisco, CA., pp: 246-610. DOI: 10.1109/ISSCC.2008.4523149

Aigner, R., 2005. MEMS in RF-filter applications: Thin film bulk-acoustic-wave technology. Proceedings of the The 13th International Conference on SolidState Sensors, Actuators and Microsystems, Jun. 5-9, IEEE Xplore Press, Germany, pp: 5-8. DOI: 10.1109/SENSOR.2005.1496345
Bi, F.Z. and B.P. Barber, 2008. Bulk acoustic wave RF technology. IEEE Microwave Mag., 9: 65-80. DOI: 10.1109/MMM.2008.927633

Flatscher, M., M. Dielacher, T. Herndl, T. Lentsch and R. Matischek et al., 2009. A robust wireless sensor node for in-tire-pressure monitoring. Proceedings of the IEEE International Solid-State Circuits Conference, Feb. 8-12, IEEE Xplore Press, San Francisco, CA., pp: 286-287. DOI: 10.1109/ISSCC.2009.4977420

Flatscher, M. M. Dielacher, T. Herndl, T. Lentsch and R. Matischek et al., 2010. A Bulk Acoustic Wave (BAW) based transceiver for an in-tire-pressure monitoring sensor node. IEEE J. Solid-State Circ., 45: 167-177. DOI: 10.1109/JSSC.2009.2034436

Gruber, S., H. Reinisch, H. Unterassinger, M. Wiessflecker and G. Hofer et al., 2010. A passively powered BAW-based multi-standard intire identification and monitoring system. Proceedings of the 7th International Symposium on Communication Systems Networks and Digital Signal Processing, Jul. 21-23, IEEE Xplore Press, Newcastle Upon Tyne, pp: 300-304.

Missoni, A., C. Klapf, W. Pribyl, H. Guenter and G. Holweg, 2008. A triple-band passive RFID tag. Proceedings of the IEEE International Solid-State Circuits Conference, Feb. 3-7, IEEE Xplore Press, San Francisco, CA., pp: 288-614. DOI: 10.1109/ISSCC.2008.4523170

Peng, Z., Y. Wei, Z. Ke and L. Wenhong, 2009. An offset-insensitive switched-capacitor bandgap reference with continuous output. J. Semicond., 30: 085006-085006. DOI: $10.1088 / 1674-$ 4926/30/8/085006

Reinisch, H., S. Gruber, M. Wiessflecker, H. Unterassinger and G. Hofer et al., 2010. An electro-magnetic energy harvester with $190 \mathrm{nW}$ idle mode power consumption for wireless sensor nodes. Proceedings of the ESSCIRC, Sept. 14-16, IEEE Xplore Press, Sevilla, pp: 234-237. DOI: 10.1109/ESSCIRC.2010.5619886 